Artículo

\title{
La política pública hacia la agricultura protegida en el gobierno de López Obrador
}

\author{
José Luis Hernández Suárez ${ }^{\S}$ \\ Unidad Académica de Ciencias Sociales-Campus Universitario II-Universidad Autónoma de Zacatecas. Av. \\ Preparatoria s/n, Fraccionamiento Progreso, Zacatecas, Zacatecas, México. CP. 9800. \\ ${ }^{\S}$ Autor para correspondencia: jels_hs@uaz.edu.mx.
}

\section{Resumen}

Durante el gobierno de Vicente Fox Quesada se implementó una política pública orientada al desarrollo de la agricultura protegida, en el marco de la estrategia para aumentar la competitividad del sector agrícola que es fuente de divisas y para complementar a las economías norteamericana y canadiense en el abasto de hortalizas y plantas de ornato. Creció la superficie, el número de estructuras y la producción a partir de entonces, pero en el gobierno de Andrés Manuel López Obrador, los productores agrícolas empresariales perdieron poder para dirigir la política en beneficio de sus intereses, como consecuencia de cambios en la correlación de fuerzas. El objetivo de este trabajo fue analizar cómo fue desaparecida dicha política durante el año 2020, con base en una perspectiva crítica del conflicto entre los grupos sociales por avanzar sus asuntos y bloquear los proyectos de sus rivales. El estudio se apoya en textos sobre producción e información de posturas de asociaciones de productores y del gobierno. Los resultados son una finalización de los apoyos a la producción y comercialización de la agricultura protegida.

Palabras clave: cambio de política pública agrícola, lucha de intereses en la política pública agrícola, presupuesto para la agricultura.

Recibido: julio de 2021

Aceptado: septiembre de 2021 


\section{Introducción}

Desde su comienzo en 2018, el gobierno de Andrés Manuel López Obrador, con énfasis en el ejercicio de un gasto austero y combate a la corrupción, comenzó a cuestionar diversos programas de gobiernos anteriores, como el Programa 3x1 para migrantes, el Programa de Estancias Infantiles y una gran cantidad de fideicomisos, hasta desaparecerlos o reducir los recursos presupuestales, la plantilla laboral y su estructura.

A su periodo el presidente López Obrador lo definió como la Cuarta Transformación, entendida como una transformación pacífica contra la corrupción y la impunidad con un modelo posneoliberal basado en la economía moral, una democracia participativa y la separación del poder político y el poder económico (López, 2019). Los proyectos prioritarios vendrían a ser el sostenimiento y ampliación de transferencias monetarias a adultos mayores, jóvenes, estudiantes, el fortalecimiento de Pemex y la Comisión Federal de Electricidad, la construcción de la Refinería 'Dos Bocas' en Tabasco, el Tren Maya en el sureste del país, la edificación del Aeropuerto Internacional 'Felipe Ángeles' en la Base Aérea Militar número 1 en el Estado de México, el Tren de Alta Velocidad México-Querétaro, por mencionar algunos de los más emblemáticos de su gestión. Además de estar dirigida contra el régimen neoliberal, el propósito es que dicha trasformación pacífica dé lugar a un régimen diferente.

López Obrador obtuvo 54.8\% de los votos válidos en el 2018, ganó en 31 de los 32 estados y en 267 de los 300 distritos federales, un 'tsunami' electoral (Sonnleitner, 2020), logrado gracias a la aplicación de una estrategia de comunicación diversificada enfocada en la personalización de la política (Hernández, 2019) y la insatisfacción ciudadana con el funcionamiento de la democracia y la economía, que percibía, de manera mayoritaria, que el país estaba gobernado por unos cuantos grupos poderosos para su beneficio (Latinobarómetro, 2018). Todo esto hizo posible aprovechar la fuerza de mayoría en el congreso para aprobar una redistribución del ingreso de acuerdo con los proyectos prioritarios del gobierno (Bartra, 2019).

En los periodos anteriores, los grupos empresariales poderosos habían disfrutado de un mayor acceso al poder estatal, gracias a la entronización de élites políticas aperturistas enfocadas en el mercado internacional ante el estrechamiento del mercado interno (Salas, 2014), lo cual se materializó en ventajas normativas, fiscales, comerciales, pero también en una retórica oficial favorable y una fuerte propagación de su ideología, si bien bajo un ambiente de consenso político conflictivo (Hernández, 2014).

El gobierno de López Obrador se orientó hacia una mayor atención a grupos vulnerables pobres y marginados, pequeños empresarios, asalariados de bajos ingresos, comunidades indígenas y campesinas. Pero distintas asociaciones de empresarios presionaron por el cambio de políticas en un sentido que les beneficiara. En ese marco se plantean las siguientes cuestiones: ¿de qué maneras fue afectada la agricultura protegida por las políticas de la Cuarta Transformación? ¿cuáles fueron las reacciones de los empresarios de este subsector? ¿por qué en el gobierno de Andrés Manuel López Obrador los productores agrícolas empresariales perdieron poder para dirigir la política pública hacia la agricultura protegida, en beneficio de sus intereses? ¿por qué hubo cambios en la correlación de fuerzas? ¿a favor y en contra de quiénes se dieron dichos cambios? y ¿por qué finalizaron los apoyos a la producción y comercialización de la agricultura protegida? 
La hipótesis de este trabajo es que, debido a la pérdida de fuerza de los intereses de los grupos empresariales agrícolas en el gobierno de Andrés Manuel López Obrador, la política pública hacia la agricultura protegida llegó a una fase de terminación, evidenciada en la eliminación de los apoyos en el presupuesto federal, en el cual se priorizaron los proyectos del nuevo gobierno. El objetivo de este trabajo fue analizar la desaparición de la política pública para la agricultura protegida durante el año 2020, con base en un planteamiento crítico del conflicto entre los grupos de interés por avanzar sus asuntos y bloquear los proyectos de sus rivales en el ámbito público, desde la perspectiva del ciclo de las políticas públicas.

Es de interés revisar dicha perspectiva porque entre las fases se plantea la posibilidad de la terminación de una política, asunto que merece explicación y para ello puede ayudar una consideración de las relaciones entre el ámbito económico, el de gobierno y el normativo, así como el análisis de las contradicciones por las luchas de intereses, que se expresan en la gestión estatal. En términos metodológicos, con base en las etapas de las políticas públicas, información oficial y de asociaciones de productores, se hace una revisión del crecimiento de la agricultura protegida y de sus cambios en la política pública. Por esa ruta, se intenta comprender las reacciones de los intereses afectados por la terminación de la política bajo el gobierno de la Cuarta Transformación y la relación de esta gestión con la agricultura protegida. Al final se presentan las conclusiones del análisis.

\section{Materiales y métodos}

\section{Entre la gestión pública y la gestión privada}

Una cuestión básica en la gestión pública, visto de manera abstracta, tiene que ver con la presencia de acotamientos que socavan su efectividad, ya que no es un asunto de carácter tecnocrático o administrativo solamente sino también y principalmente, económico y político. El marxismo ha destacado las contradicciones inmanentes al desarrollo del capital y la lucha de clases que le es inherente, por lo cual la acumulación requiere de la gestión estatal para hacer manejables dichas contradicciones, al menos durante ciertos periodos de tiempo, el carácter de la gestión define determinados tipos de regímenes de acumulación (Chesnais, 2003), para hacer manejables las tendencias autoparalizantes del capital.

Esta forma de ver las cosas supone un Estado separado de la economía, pero en realidad el Estado se encuentra inmerso en las propias relaciones económicas (Offe, 2015) y cumple funciones propicias y adecuadas a la acumulación (Figueroa, 1995). Desde otro punto de vista de la teoría social crítica, la gestión pública remite a la consideración de procesos de orden económico, político-administrativo y normativo (Offe, 1990).

La gestión estatal se encuentra atravesada por contradicciones propias de la universalidad que le es exclusiva en las sociedades capitalistas, ya que debe articular las demandas de intereses contradictorios, por una parte y por otra, porque en ella recaen las deficiencias de los procesos administrativos y las tareas encaminadas a lograr consensos, lo cual hace que el Estado se presente como un poder superior para asegurar la reproducción social capitalista (Boundi, 2020). 
Sin embargo, las demandas rebasan la capacidad de respuesta del sistema de gobierno y sus instituciones político-administrativas, de manera que la inconformidad y el conflicto están garantizados, ya que, por una parte, la gestión privada de la producción y la tendencia de las empresas a mercantilizar todo aquello que les reditúe ganancias, se contrapone con las presiones de los sectores sociales que buscan desmercantilizar la satisfacción de sus necesidades. Desde la perspectiva de la teoría de la democracia capitalista, las imposibilidades de avanzar más allá de cierto punto tienen que ver con el conflicto irreconciliable entre la eficiencia marginal del capital y la realización de los intereses de los grupos sociales (Figueroa, 2018).

Estas luchas de intereses se expresan en la politización del proyecto y la materia de la función de gobierno, esto es, las políticas públicas (Vilas, 2011; Guerrero, 2014), en todas y cada una de sus fases: puesta de la agenda pública, definición del problema, formulación de la política, implementación, evaluación, redefinición y/o terminación (Jann y Wegrich, 2007; Birkland, 2007; Sydney, 2007; Pülzl y Treib, 2007; Wollmann, 2007).

Si bien el poder de los contendientes para influir en cada una de las etapas del ciclo es variable, la lucha subyace a todas las fases y también, puesto que las refriegas se dan en el marco de un Estadonación, desde una perspectiva de gobernanza, los contendientes 'se necesitan' (Aguilar, 2006) y colaboran para reducir las fallas de la implementación y los altos costos de la politización de la regulación (Ansell y Gash, 2008). Por eso el análisis de las políticas públicas requiere 'una comprensión profunda de los procesos políticos' (Bustelo, 2011).

Desde la puesta de la agenda pública, los grupos buscan que entren sus demandas y tratan de que se excluyan aquellas de otros grupos que van en sentido contrario a las suyas o que tienden a dificultar su satisfacción plena. En esas luchas, tratan de que sus demandas sean reconocidas como problemas y definidos en los términos que a ellos conviene, procurando influir la opinión pública para tenerla a su favor (Williamson y Luke, 2020), lo cual suele no ser fácil, pues a cada paso se ven impugnados por grupos rivales y afectados, además por las inercias de las estructuras administrativas y el conjunto de sus recursos disponibles.

Los esfuerzos por empujar los asuntos y lograr su reconocimiento todavía deben recorrer un camino bastante difícil. El problema tiene que recibir propuestas de alternativas de solución y ha de hacerse una formulación que será implementada, no siempre con los resultados deseados, porque la lucha entre los intereses y valores rivales permea todo el ciclo de la política pública. Las políticas públicas son objeto de evaluación y escrutinio de distintos tipos, en mayor o menor grado y pueden continuar, de acuerdo con las correlaciones de fuerza, posiblemente reformuladas o bien, es factible que los grupos políticos a quienes buscaba beneficiar esa política pierdan la lucha frente a otros contendientes en un cambio de condiciones y correlaciones de fuerza, de modo que la política pública en cuestión puede ser terminada o debilitada hasta reducirla a la insignificancia.

La terminación es de las menos estudiadas; sin embargo, 'la política pública establecida para afrontar un problema puede terminar, lo que en sentido estricto se entendería como que abandona la agenda estatal, ya sea para desaparecer por completo o para regresar a ser un problema o cuestión social' (Méndez, 2020). Un grado de incertidumbre impregna a las políticas públicas en cada una de sus fases, pero cuando las correlaciones llevan a cambios radicales que provocan sucesiones de poder central con una alta concentración de fuerza, se dan redefiniciones según el estado de la lucha de intereses. Pueden concurrir varios motivos para la terminación de política, pero la 'falta de 
acuerdo político’ es uno de los más comunes y directos (Méndez, 2020). Enseguida, se tratarán de comprender las circunstancias por las cuales la agricultura protegida tomó fuerza en México y fue objeto de las acciones de gobierno, así como la tendencia a su caída de la consideración pública.

\section{Resultados y discusión}

\section{Auge de la agricultura en México y su gestión pública}

La agricultura protegida es aquella realizada bajo clima controlado o relativamente controlado, con estructuras plásticas, metálicas y vidrios, equipada de tal forma que suministra el agua y nutrientes de maneras más eficientes y optimiza los insumos, materiales y mano de obra para obtener más altos rendimientos de la producción con mejor calidad y que puede efectuarse sin seguir la estacionalidad de la agricultura a cielo abierto. En México, mayormente se dedica al cultivo de hortalizas, algunos tipos de frutas y plantas ornamentales (Figura 1).

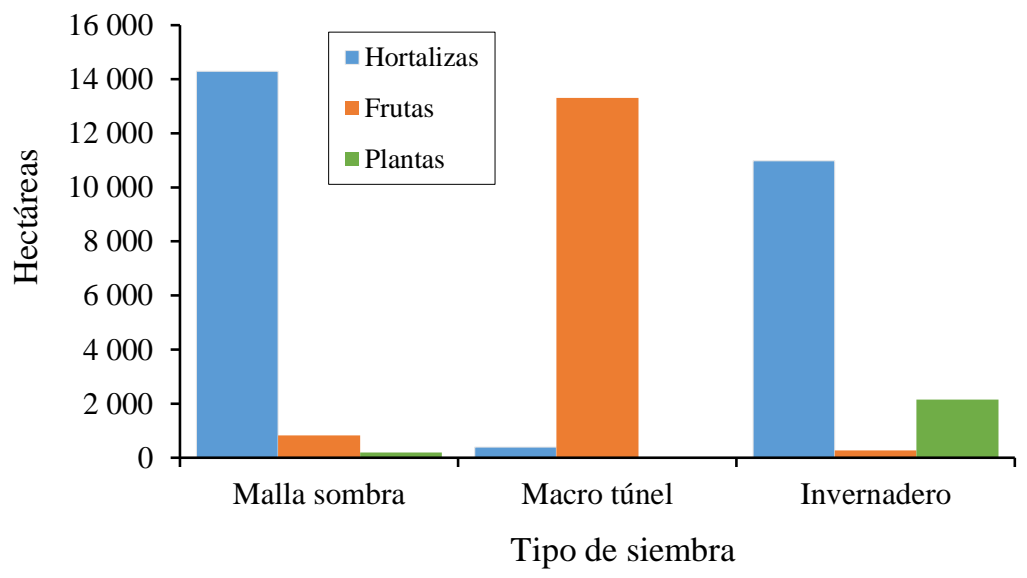

Figura 1. Superficie de agricultura en México 2017 (AMHPAC, 2018).

Como puede suponerse, una agricultura de esta clase requiere de fuertes sumas de inversión, dependiendo del tipo de estructura de que se trate, por lo cual es efectuada básicamente por productores u organizaciones de productores de tipo empresarial, una parte de ellos con orientación a la exportación. Con el avance del capital en la agricultura, la de tipo protegido comenzó a utilizarse a mediados del siglo XX y desde principios del XXI se expandió rápidamente por varias regiones del país. En la actualidad se practica en la mayoría de las zonas agrícolas de México, pero en términos de superficie se encuentra más concentrada en pocas entidades, principalmente en Sinaloa, Jalisco y Michoacán, que suman 56\% de la superficie, seguidos por Sonora, Baja California y San Luis Potosí, el Estado de México y Guanajuato (AMHPAC, 2018).

La superficie destinada a la agricultura protegida pasó de 132 ha en 2003 a 4877 en 2009 y hacia el 2017 se habían alcanzado 42515 ha. Únicamente en los años 2008, 2013 y 2015 hubo reducción de superficie sembrada respecto al año anterior, pero continuó la tendencia al alza (Figura 2). Para desarrollarse de esa manera, esta forma de agricultura tuvo condiciones de mercado propicias, un medio ambiente social favorable y una gestión gubernamental adecuada, que ayudó al el éxito y expansión de este tipo de negocios de producción y comercialización de alimentos y plantas ornamentales. 


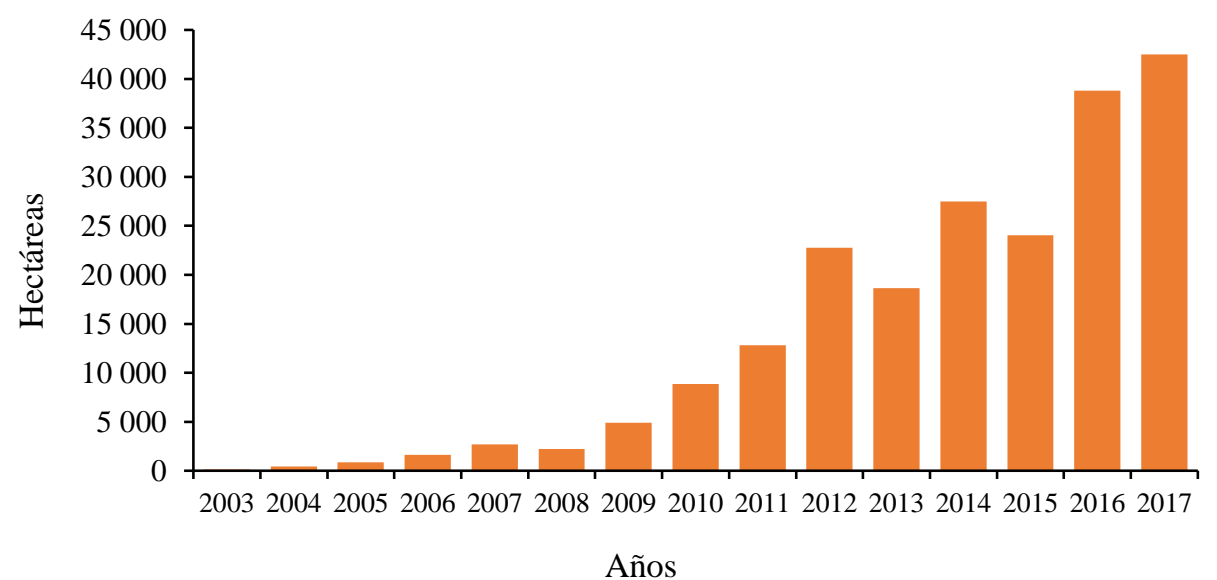

Figura 2. Superficie sembrada de agricultura protegida, 2003-2017 (AMHPAC, 2018).

El inicio y crecimiento de la agricultura protegida en el país no se dio de manera aislada. La demanda de hortalizas por parte de Estados Unidos de América y Canadá en momentos del año en que esos países tienen mayores dificultades de orden climático para producirlas, explica el rol de México en ese rubro, al amparo de la integración comercial regulada por el Tratado de Libre Comercio de América del Norte, firmado en 1994 por los presidentes de los tres países, ya que este tipo de agricultura está enfocada, en gran medida, a la exportación, al menos la de los grandes empresarios de este subsector y principalmente en tres productos: tomates, pepinos y pimientos morrones (Figura 3), si bien la producción se ha ido diversificando a muchos más productos, pero sin alcanzar la importancia de los que se mencionan.

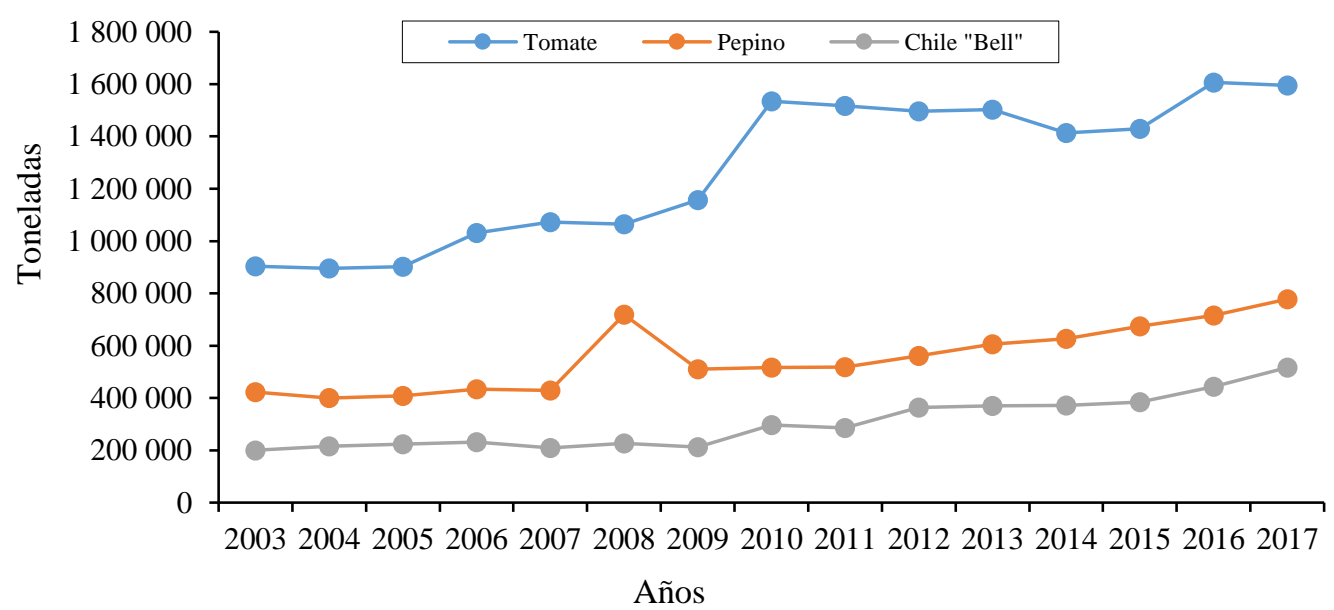

Figura 3. Volumen de exportaciones de tomate, pepino y pimientos morrones 2003-2017 (AMHPAC, 2018).

La llegada de Vicente Fox Quesada a la presidencia de la república (2000-2006) conllevó una mayor presencia de grupos empresariales en la gestión estatal y con él comenzó el acceso de presidentes empresarios al poder en América Latina en este siglo, sin intermediarios (Nercesian, 2021). Diversas secretarías y puestos importantes fueron ocupados por empresarios, personajes surgidos del sector privado, educados en universidades nacionales y extranjeras con orientación empresarial. 
Desde la Secretaría de Agricultura, Ganadería, Pesca y Alimentación (SAGARPA), a cargo de Javier Usabiaga, un gran productor de ajo, se impulsaron programas de apoyo para el establecimiento de estructuras de agricultura protegida, por lo que la superficie de esta comenzó a crecer, como ya se ha dicho. El objetivo era propiciar el avance del capital en la agricultura para aumentar los volúmenes de exportaciones y favorecer la balanza comercial del país.

Durante el gobierno de Fox Quesada se enfatizó un perfil de productor de tipo empresarial, con capital y capacidades para implantarse, sostenerse y crecer, incluso sin necesidad de los apoyos de gobierno, pero a la vez estos se dirigieron a una gran cantidad de productores que no contaban con recursos y no podrían permanecer en el medio, causa por la cual creció el número de estructuras de agricultura protegida pero concentradas en menos de una hectárea y con un aumento de los proyectos abandonados o inactivos (Hernández, 2020).

En los gobiernos de Felipe Calderón Hinojosa (2006-2012) y Enrique Peña Nieto (2012-2018) la agricultura protegida siguió disponiendo de apoyo presupuestal, básicamente para instalación y mantenimiento de las estructuras, si bien con Peña Nieto los recursos se fueron reduciendo (Hernández, 2020). Vista a través de la perspectiva del ciclo de las políticas públicas, la agricultura protegida subió a la agenda de la política pública con la llegada directa de los empresarios al poder, a partir del gobierno de Vicente Fox en el año 2000 y hasta 2018 permaneció en el ámbito de la política pública agrícola.

\section{La cuarta transformación y la agricultura protegida}

La persistencia de la desigualdad en la distribución del ingreso en el país (Cuadro 1) y los altos niveles de pobreza, más allá de las divergencias sobre sus tipos y niveles (Figura 4), aunado al desgaste de los gobiernos anteriores, hicieron que López Obrador replanteara o terminara varios programas y políticas.

Cuadro 1. Coeficiente de Gini y distribución del ingreso corriente total de los hogares por quintil, con y sin ajuste a Cuentas Nacionales, México, 2016.

\begin{tabular}{ccc}
\hline Coeficiente & Sin ajuste a CN & Con ajuste a CN \\
\hline Gini nacional & 0.463 & 0.588 \\
Gini CDMX & 0.439 & 0.542 \\
I & \multicolumn{2}{c}{$(\%)$ de ingreso por quintil } \\
II & 4.8 & 4.3 \\
III & 9.1 & 7.3 \\
IV & 13.7 & 10.4 \\
V & 20.6 & 14.7 \\
& 51.8 & 63.2 \\
\end{tabular}

Damián (2019). 


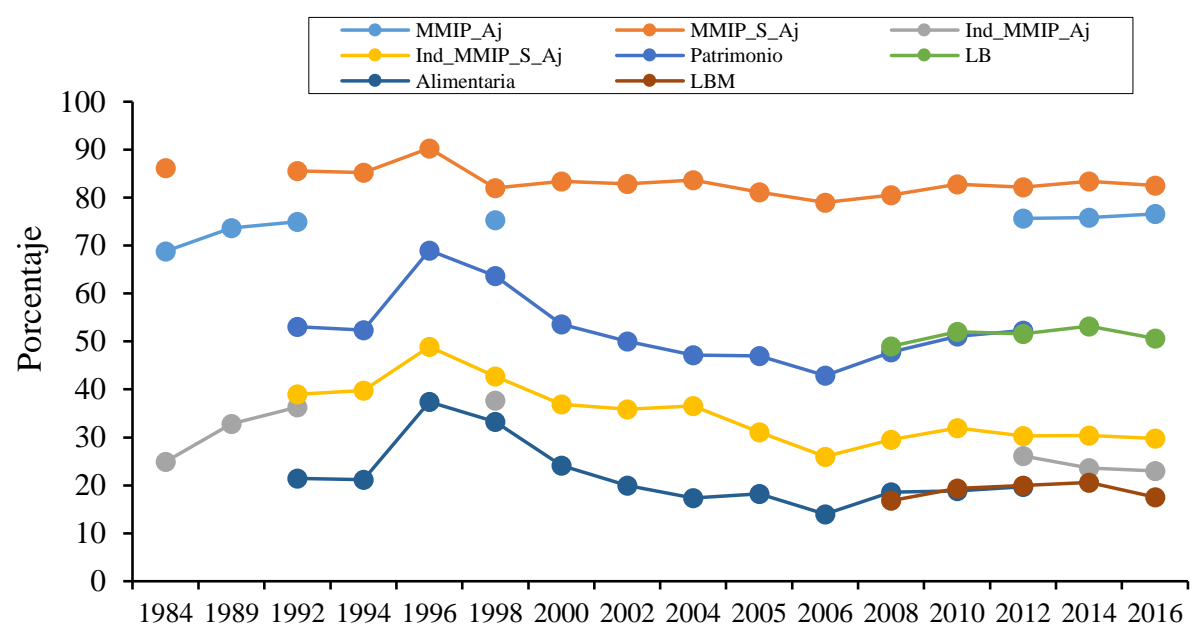

Años

Figura 4. Evolución de la pobreza en México, varios métodos (Damián, 2019).

Se dejó de suministrar apoyos para la instalación y reposición de plásticos de la agricultura protegida, también se redujo el crédito y el financiamiento público. En el proceso de definición del presupuesto de 2019 se dieron acalorados debates y diversas manifestaciones contra el gobierno, por parte de quienes vieron menguados sus apoyos.

Ante la propuesta de la Secretaría de Hacienda y Crédito Público (SHCP), de reducir el presupuesto de la Secretaría de Agricultura y Desarrollo Rural (SADER) en el 2020 a 46253.1 millones de pesos -había contado con 65434.9 millones en el 2019- el Congreso Agrario Permanente, el Consejo Nacional Agropecuario, la Confederación Nacional de Propietarios Rurales, la Confederación Nacional de Organizaciones Ganaderas y la Confederación Nacional Campesina, se inconformaron mediante un escrito de diez puntos, de los cuales cabe resaltar los siguientes cinco como los más importantes.

La necesidad de contar no sólo con programas sociales que contribuyen a superar las condiciones de pobreza y marginación, sino también con programas productivos que fortalezcan la inclusión productiva. La posibilidad de que haya una pronunciada caída de la producción agropecuaria y un desequilibrio en la balanza comercial agropecuaria, así como una disminución en los ingresos de los productores rurales, el deterioro de las condiciones de vida y la estabilidad social en el medio rural.

La necesidad de incrementar el presupuesto de los programas productivos del campo para reactivar la economía, el mercado interno y el empleo. La importancia de no reducir el presupuesto al campo, ya que se correría el riesgo de pasar de un Estado mínimo a un Estado ausente y generador de pobreza. Las bondades de arraigar a la población rural y reconstruir el tejido social por medio de financiamiento, asistencia técnica, capacitación y apoyos al cultivo, producción y comercialización, medidas sanitarias y aseguramiento (La Jornada del Campo, 2019). El comparativo entre la propuesta de la SHCP y lo aprobado en el 2019 se muestra en el (Cuadro 2). 
Cuadro 2. Presupuesto de la Secretaría de Agricultura y Desarrollo Rural en 2019 y 2020.

\begin{tabular}{|c|c|c|c|}
\hline Programa & $\begin{array}{c}\text { PEC aprobado } \\
2019 \\
\text { (millones de pesos) }\end{array}$ & $\begin{array}{c}\text { Propuesta SHCP } \\
2020 \\
\text { (millones de pesos) }\end{array}$ & $\begin{array}{l}\text { Variación } \\
\text { porcentual }\end{array}$ \\
\hline Agromercados sociales y sustentables & 6708 & - & -100 \\
\hline Crédito Ganadero a la Palabra & 4000 & 1000 & -75 \\
\hline Fertilizantes & 1500 & 1000 & -33 \\
\hline Desarrollo Rural & 5375 & 70 & -99 \\
\hline Capitalización Productiva Agrícola & 763 & - & 100 \\
\hline $\begin{array}{c}\text { Estrategias Integrales de Políticas Públicas } \\
\text { Agrícolas }\end{array}$ & 262 & - & 100 \\
\hline $\begin{array}{c}\text { Investigación, Innovación y Desarrollo } \\
\text { Tecnológico Agrícola }\end{array}$ & 987 & - & 100 \\
\hline Mejoramiento Productivo de Suelo y Agua & 731 & - & 100 \\
\hline Impulso a la Capitalización & 978 & - & 100 \\
\hline Paquetes Productivos Pesqueros y Acuícolas & 58 & - & 100 \\
\hline $\begin{array}{c}\text { Programa de Concurrencia con las Entidades } \\
\text { Federativas }\end{array}$ & 2000 & - & 100 \\
\hline Programa de Fomento Ganadero & 500 & - & 100 \\
\hline Acceso al Financiamiento & 424 & - & 100 \\
\hline Activos Productivos y Agrologística & 356 & - & 100 \\
\hline $\begin{array}{c}\text { Certificación y Normalización } \\
\text { Agroalimentaria }\end{array}$ & 13 & - & 100 \\
\hline $\begin{array}{l}\text { Desarrollo Productivo del Sur Sureste y } \\
\text { Zonas Económicas Especiales }\end{array}$ & 331 & - & 100 \\
\hline Fortalecimiento a la Cadena Productiva & 77 & - & 100 \\
\hline Riesgo Compartido & 99 & - & 100 \\
\hline $\begin{array}{c}\text { Programa de Sanidad e Inocuidad } \\
\text { Agroalimentaria }\end{array}$ & 4128 & 3749 & -9 \\
\hline $\begin{array}{c}\text { Sistema Nacional de Información para el } \\
\text { Desarrollo Rural Sustentable }\end{array}$ & 104 & - & 100 \\
\hline Sistema Nacional de Investigación Agrícola & 35 & - & 100 \\
\hline Desarrollo Rural & 844 & 10 & -99 \\
\hline Energías Renovables & 145 & - & 100 \\
\hline $\begin{array}{c}\text { Programa de Fomento a la Productividad } \\
\text { Pesquera y Acuícola }\end{array}$ & 31 & - & 100 \\
\hline $\begin{array}{l}\text { Adquisición de leche a productores } \\
\text { nacionales }\end{array}$ & 1769 & - & 100 \\
\hline
\end{tabular}

La Jornada del Campo (2019).

Finalmente, el presupuesto aprobado para la SADER se mantuvo casi como lo propuso la SHCP. La Cuarta Transformación aprovechó su fuerza de mayoría en el Congreso y se aprobaron 47576 millones de pesos (Cámara de Diputados, 2019). La Subsecretaría de Alimentación y 
Competitividad de la SADER elaboró un documento con un diagnóstico y las razones para el cambio de política (Cuadro 3), en el cual argumentó que para reducir la pobreza y la desigualdad social se tenían que implementar políticas diferentes. Sobre los opositores, escribió que: pretenden dar una idea de que cuando se contó con presupuestos elevados para el campo, los indicadores económicos y sociales del sector eran 'buenos' y que en el PPEF 2020 se 'abandona' a los productores rurales a su suerte [...] es una falacia tal la fatalidad augurada para 2020. Los indicadores socioeconómicos que se conocen del sector y los resultados obtenidos manifestaron una tendencia diferente e incluso contraria respecto de los crecientes presupuestos que se ejercieron durante 2003-2018 (SADER, 2019).

\section{Cuadro 3. Diagnóstico y razones de la SADER para el cambio en la política pública para el campo.}

El ingreso de los productores se encuentra El crecimiento de los rendimientos por cultivo es estancado escaso

La balanza comercial está desacoplada del presupuesto asignado a la SADER

Existe dependencia alimentaria de cultivos básicos y estratégicos

El sector primario tiene un crecimiento menor respecto al crecimiento de toda la economía

Los subsidios productivos se concentraron y aumentaron las desigualdades entre regiones y productores

El ochenta por ciento del presupuesto estaba asignado a bienes privados individuales y no a bienes públicos que estimularan las acciones del conjunto de la población
El impacto de los apoyos productivos era bajo o nulo

La productividad en el sector agrícola se encuentra estancada

Existía un escaso acceso a programas de carácter productivo

Las Reglas de Operación de los programas eran una barrera para el acceso a los apoyos y la articulación

Había una alta captura de los apoyos por parte de diversos actores para focalizar los recursos a favor de sus intereses y agrupaciones, sin dispersarlos hacia las regiones productivas

Elaboración con base en la SADER (2019).

El Programa Especial Concurrente para el Desarrollo Rural, como se había venido aplicando en los gobiernos anteriores, de acuerdo con la SADER, en resumidas cuentas: no generó mejores condiciones socioeconómicas en el campo por su orientación a bienes privados individuales, su regresividad, que aumenta las desigualdades regionales y entre productores; el desacoplamiento de las distintas estrategias de intervención, lo que genera duplicidad o que las intervenciones se anulen entre si, la captura de los recursos por ciertos sectores de la sociedad rural, que genera altos costos de transacción y que una parte del recurso público se quede en el camino; el excesivo número de programas que pulverizan la acción gubernamental, la falta de claridad en las poblaciones objetivo y en la focalización, lo que genera dispersión y que se atienda a productores que no presentan el problema y el elevado burocratismo para acceder a los apoyos, que se expresa en las Reglas de Operación (SADER, 2019).

De esta manera se terminaba el 'apoyo decisivo del gobierno mexicano' en la forma y términos en que se había dado para la agricultura empresarial (De Ita, 2014), la cual, a lo largo de los años se benefició de inversión pública en servicios e infraestructura, mejores tierras, mayor acceso al gobierno de forma organizada y una narrativa favorable en el Estado (Turrent, 2018). 
A causa de la reducción radical de los apoyos, uno de los líderes del Consejo Nacional Agropecuario lamentó: 'desaparecen los recursos fiscales para FIRA, FND, FOCIR y para Agroasemex se reduce 50\%, por lo que no solo se disminuyen los apoyos al sector productivo, sino también aquellos que puede otorgar la banca de desarrollo en materia de financiamiento y seguros' (Haro, 2019). La fuerte lucha comenzó a darse en la definición del presupuesto de 2019 en la Cámara de Diputados, cuando organizaciones campesinas, empresariales, gobernadores, 'grupos de presión que año con año van a reclamar lo suyo' presionaron para no quedar fuera de los apoyos (Bartra, 2019). Pero se impuso la fuerza mayoritaria del grupo en el poder y en el Presupuesto de Egresos de la Federación se programaron 49291 millones de pesos (Cámara de Diputados, 2020).

Se redujo el presupuesto para la agricultura empresarial y dentro de ella, la agricultura protegida. Sin embargo, los empresarios siguieron presionando para que el gobierno reconsidere la política hacia el sector. Por ejemplo, ante la crisis sanitaria por causa del SARS-CoV-2 que provoca la enfermedad de COVID-19, el 14 de octubre de 2020 la Asociación Mexicana de Horticultura Protegida (AMHPAC) publicó un texto en el que los productores de frutas y hortalizas de Ontario, Canadá, manifestaron su complacencia 'con el anuncio de financiación conjunta del gobierno federal y provincial de $\$ 11.6$ millones adicionales para apoyar aún más a los productores en sus esfuerzos por proteger a los trabajadores agrícolas durante la pandemia de COVID-19' (AMHPAC, 2020). Es una forma de comparar el apoyo que reciben los productores de otro país, frente al que en México no se les da.

No obstante, las críticas el gobierno respaldó a los exportadores agroalimentarios mexicanos de otras maneras. Por ejemplo, a mitad del año 2019 el presidente de Estados Unidos de América amagó con imponer aranceles a las importaciones de tomate mexicano, pero el de México declaró que esa medida no favorecería la contención de la migración centroamericana (López, 2019).

La amenaza fue retirada pero los exportadores de tomate no reconocieron mérito alguno al gobierno de López Obrador, ya que en un comunicado del 19 de septiembre del mismo año, la AMHPAC mencionó la suspensión de la medida firmada por ésta, la Confederación de Asociaciones Agrícolas del estado de Sinaloa (CAADES), el Consejo Agrícola de Baja California (CABC), el Sistema Producto Tomate Nacional (SPTN) y la Asociación de Productores de Hortalizas del Yaqui-Mayo (APHYM) con el Departamento de Comercio de Estados Unidos de América, sin reconocer el respaldo del marco normativo, la firma y el respaldo político de las autoridades mexicanas (AMHPAC, 2019).

Debe decirse que en la agricultura protegida de punta en México operan no sólo capitales privados nacionales, sino también extranjeros, sobre todo de Estados Unidos de América y Canadá, altamente tecnificados y con acceso a diversas fuentes y estrategias de financiamiento, dirigidos por 'hombres de negocios y científicos', ya que 'por lo redituable que resulta la agricultura protegida se toma como un fondo de inversión y hay empresas que cotizan en bolsas de valores, a la par de que utilizan el big data y la inteligencia artificial' (González, 2020). Para los pequeños y medianos productores del subsector, básicamente orientados a los mercados del interior, el escenario es más complicado debido a problemas financieros, técnicos, administrativos y de mercadeo (Hernández 2020), que no son nuevas en el gobierno de la Cuarta Transformación, pero tampoco han desaparecido a diferencia del financiamiento. 


\section{Conclusiones}

Se han aportado elementos a la hipótesis de que la política pública hacia la agricultura protegida llegó a una fase de terminación con el gobierno de Andrés Manuel López Obrador. De acuerdo con una perspectiva crítica del ciclo de las políticas públicas, un cambio en las correlaciones de fuerzas en la gestión estatal es un factor fundamental para que se le dé finalización a una política pública, como fue el caso de la que aquí se analizó. De manera concreta, se llega a las siguientes respuestas de las preguntas formuladas en la introducción: El gobierno federal propuso la reducción de los recursos públicos para el campo a partir del presupuesto del 2020 y una reorientación de estos. En el proyecto de restricción del gasto público para el sector se eliminaron los apoyos para la agricultura protegida.

Los empresarios agrícolas reaccionaron a través de sus asociaciones y presionaron para impedir la aprobación de la medida en la cámara de diputados, pero no tuvieron la fuerza política suficiente para conseguirlo. La pérdida de poder de los empresarios agrícolas implicó el rompimiento del ciclo de la política pública hacia la agricultura protegida y la derrota de sus intereses en el ámbito presupuestal.

La reorientación de la política hacia el campo, con el debilitamiento de los intereses empresariales agrícolas en el presupuesto y la finalización de la agricultura protegida, se dio en el marco del amplio triunfo electoral del movimiento encabezado por López Obrador, en el cual se plantearon otros proyectos prioritarios y se tuvo la fuerza suficiente para avanzarlos, con la convicción de que era necesaria una reorientación de la política para reducir la pobreza, la desigualdad.

En las luchas que se dieron a partir de la segunda mitad de 2019, sobre la orientación de la política para el campo, la asignación y composición del presupuesto del sector, los productores de agricultura protegida no lograron redefinir la política pública a su favor. El gobierno federal, enfrentado a múltiples demandas, con recursos fiscales escasos y la decisión de no recurrir a más deuda, optó por la desaparición de los apoyos, aprovechando su fuerza mayoritaria en el Congreso. Es decir, se conjugaron factores de orden objetivos con decisiones políticas enfocadas a una reorientación de las acciones de gobierno.

La perspectiva crítica del ciclo de la política pública sostiene que, a lo largo de cada una de sus etapas o momentos, los grupos no dejan de luchar para empujar sus asuntos hacia la consideración de la gestión pública y con el fin de evitar que otros avancen los propios. La fuerza política del gobierno de López Obrador le permitió sostener las decisiones sobre los asuntos aquí discutidos y la política pública hacia la agricultura protegida fue terminada.

\section{Literatura citada}

Aguilar, L. 2006. Gobernanza y gestión pública. Fondo de Cultura Económica (FCE). México, DF. $500 \mathrm{p}$.

AMHPAC. 2018. Horticultura protegida en México. http://amhpac.org/2018/images/PDFoficial/ HorticulturaenMexico.pdf.

AMHPAC. 2019. Se firmó oficialmente un nuevo acuerdo de suspensión. Comunicado de prensa, 19 de septiembre. https://www.amhpac.org/es/index.php/en/informacion/comunicados/14comunicados/315-se-firmo-oficialmente-un-nuevo-acuerdo-de-suspension. 
AMHPAC. 2020. Los productores de frutas y hortalizas agradecen la financiación adicional de costos compartidos para la protección de los trabajadores agrícolas. https://www.amhpac.org/es/index.php/en/informacion/noticias/17-noticias/602-losproductores-de-frutas-y-hortalizas-agradecen-la-financiacion-adicional-de-costoscompartidos-para-la-proteccion-de-los-trabajadores-agricolas.

Ansell, C. and Gash, A. 2008. Collaborative governance in theory and practice. J. Public Adm. Res. Theory. 18(4):543-571. https://doi.org/10.1093/jopart/mum032.

Bartra, A. 2019. Un presupuesto para el cambio. La Jornada del Campo (136). https://www.jornada.com.mx/2019/01/19/cam-presupuesto.html.

Birkland, T. 2007. Agenda setting in public policy. In: Handbook of public policy analysis. Theory, politics and methods. Fischer, F. (Ed.). CRC press. New York 63-78 p. https://pdfs.semanticscholar.org/bcf4/94ee9d9b8b715c876006447f90b1210e7c09.pdf.

Boundi, F. 2020. El Estado y la sociedad en la economía política. Rev. Econ. Instituc. 22(42):187210. https://doi.org/10.18601/01245996.v22n42.08.

Bustelo, M. 2011. Evaluación en el marco del análisis de políticas públicas. In: evaluación de políticas públicas. Salcedo, R. (coord.). Siglo XXI. México, DF. 185-252 p.

Cámara de Diputados. 2019. Presupuesto de egresos de la federación para el ejercicio fiscal 2020. http://www.diputados.gob.mx/LeyesBiblio/ref/pef_2020/PEF_2020-orig_11dic19.pdf.

Cámara de Diputados. 2020. Presupuesto de egresos de la federación para el ejercicio fiscal 2021. http://www.diputados.gob.mx/LeyesBiblio/pdf/PEF_2021-301120.pdf.

Chesnais, F. 2003. La teoría del régimen de acumulación financiarizado: contenido, alcances e interrogantes. Rev. Economía Crítica (1):37-72. http://www.revistaeconomiacritica.org/ sites/default/files/revistas/n1/4_teoria_regimen.pdf.

Damián, A. 2019. Pobreza y desigualdad en México. La construcción ideológica y fáctica de ciudadanías diversas y desiguales. El trimestre económico 86(343):623-666. https://doi.org/10.20430/ete.v86i343.920.

De-Ita, A. 2014. México: Economía campesina y agricultura empresarial veinte años después. Alasru. 9:53-81. http://ceccam.org/sites/default/files/Revista\%20ALASRU.pdf.

Figueroa, V. 1995. La gestión estatal del desarrollo en América Latina. Problemas del Desarrollo. 26(103):129-165. http://dx.doi.org/10.22201/iiec.20078951e.1995.103.29278.

Figueroa, V. 2018. Desarrollo y democracia: teoría y actualidad en Estados Unidos (EU) de América. Perspectivas Sociales. 20(1):7-33. http://perspectivassociales.uanl.mx/index.php/ pers/article/view/36.

González, S. 2020. Rendimiento de cultivo protegido supera al menos cinco veces el de campo abierto. La Jornada, 9 de febrero. https://www.jornada.com.mx/2020/02/09/economia/ $014 \mathrm{n} 2 \mathrm{eco}$.

Guerrero, O. 2014. ¿Qué es la política pública? Notas para un marco conceptual. Examen. 23(227):7-13. http://www.omarguerrero.org/articulos/queespoliticapublica.pdf.

Haro, L. 2019. La propuesta de presupuesto olvidó la agricultura comercial. La Jornada del Campo. 145. https://www.jornada.com.mx/2019/10/19/Images/delcampo145.pdf.

Hernández, C. 2019. Campañas electorales presidenciales pragmáticas en México 2018. Política y comunicación. Rev. Mex. Cienc. Polít. Soc. 64(235):327-352. http://dx.doi.org/10.22201/ fcpys.2448492xe.2019.235.67468.

Hernández, J. 2020. El desarrollo de los invernaderos en Zacatecas. Entre el auge y el abandono. Entreciencias. 8(22):1-15. http://dx.doi.org/10.22201/enesl.20078064e.2020.22.70248. 
Hernández, R. 2014. Entre la racionalidad tecnocrática y la gobernabilidad. La importancia del consenso político en México. Rev. Mex. Cienc. Polít. Soc. 59(222):353-368. http://www.revistas.unam.mx/index.php/rmcpys/article/view/47736/42924.

Jann, W. and Wegrich, K. 2007. Theories of the policy cycle. In: handbook of public policy analysis. theory, politics and methods. Fischer, F. (Ed). CRC pres. New York. CRC press. New York. 43-62 pp. https://pdfs.semanticscholar.org/bcf4/94ee9d9b8b715c876006447 f90b1210e7c09.pdf.

La Jornada del Campo. 2019. El ajuste presupuestal significa pasar de un Estado mínimo a uno ausente y generador de pobreza. Se inconforman los agroempresarios y la CNC. La Jornada del Campo. 145. https://www.jornada.com.mx/2019/10/19/Images/delcampo145.pdf.

Latinobarómetro. 2018. Resultados por sexo y edad. Estudio LAT-20183392. 52 p. https://www.latinobarometro.org/latCodebooks.jsp.

López, A. 2019. Hacia una economía moral. Editorial Planeta. México. 192 p.

López, J. 2019. Aranceles de EU al jitomate mexicano son una medida injusta, critica AMLO. Milenio, 8 de mayo. https://www.milenio.com/negocios/amlo-aranceles-jitomate-mexicanomedida-injusta-unidos.

Méndez, J. 2020. Políticas públicas. Enfoque estratégico para América Latina. Fondo de Cultura Económica (FCE). Ciudad de México. 364 p.

Nercesian, I. 2021. Los mejores, los más preparados. Presidentes y gabinetes con sesgo empresarial en América Latina (2000-2019). Rev. Interna. Organizac. (25-26):71-102. https://doi.org/10.17345/rio25-26.71-102.

Offe, C. 1990. Contradicciones en el estado del bienestar. Editorial Alianza. México, DF. 309 p.

Offe, C. 2015. Europe entrapped. Polity press. Cambridge. 136 p.

Pülzl, H. and Treib, O. 2007. Implementing public policy. In: handbook of public policy analysis. Theory, politics and methods. Fischer, F. (Ed.). CRC press. New York. CRC press. New York. 89-107 pp. https://pdfs.semanticscholar.org/bcf4/94ee9d9b8b715c876006447f90b 1210e7c09.pdf.

SADER. 2019. Reflexiones sobre proyecto de presupuesto de 2020 para el campo mexicano. https://drive.google.com/file/d/1ciCmO11zSsldhD6jZeq-8UcueiWIwqj3/view.

Salas, A. 2014. Las élites neoliberales en México: ¿cómo se construye un campo de poder que transforma las prácticas sociales de las élites políticas? Rev. Mex. Cienc. Polít. Soc. 59(222):312-379. http://www.revistas.unam.mx/index.php/rmcpys/article/view/47733/ 42920.

Sonnleitner, W. 2020. La reconfiguración territorial de las fuerzas políticas mexicanas: geografía de la fragmentación, el colapso y la recomposición del sistema de partidos (2012-2018). Foro Internacional. 60(2):451-500. https://doi.org/10.24201/fi.v60i2.2731.

Sydney, M. 2007. Policy formulation: design and Tools. In: handbook of public policy analysis. Theory, politics and methods. Fischer, F. et al. (Ed). CRC press. New York. CRC press. New York. 79-88 pp. https://pdfs.semanticscholar.org/bcf4/94ee9d9b8b715c876006447 f90b1210e 7c09.

Turrent, A. 2018. Una SAGARPA más cercana al campo. La Jornada. 20 de julio. https://www.jornada.com.mx/2018/07/20/opinion/019a1pol.

Vilas. C. 2011. Política y políticas públicas en América Latina. In: el estado y las políticas públicas en América Latina. Fioramonti, C. y Anaya, P. (Comps.). Aecid/copppal/honorable cámara de senadores de la provincia de Buenos Aires. Buenos Aires 37-74 pp. http://cmvilas.com.ar/index.php/articulos/12-politicas-publicas/27-politica-y-politicaspublicas-en-america-latina. 
Williamson, A. and Luke, B. 2020. Agenda setting and public policy in private foundations. Nonprofit Policy Forum. 11(1):1-12. Doi: 10.1515/npf-2019-0049.

Wollmann, H. 2007. Policy evaluation and evaluation research. In: handbook of public policy analysis. Theory, politics and methods. Fischer, F. et al. (Ed.). CRC press. New York. CRC press. New York 393-402 pp. https://pdfs.semanticscholar.org/bcf4/94ee9d9b8b715c 876006447 f90b1210e7c09. 